\title{
The relationship between intelligence and anxiety: an association with subcortical white matter metabolism
}

\author{
Jeremy D. Coplan ${ }^{1}$, Sarah Hodulik ${ }^{1}$, Sanjay J. Mathew ${ }^{2}$, Xiangling Mao ${ }^{3}$, Patrick R. Hof ${ }^{4}$, Jack M. Gorman ${ }^{5}$ \\ and Dikoma C. Shungu ${ }^{3}$
}

${ }^{1}$ Division of Neuropsychopharmacology, Department of Psychiatry, State University of New York Downstate Medical Center, Brooklyn, NY, USA

${ }^{2}$ Department of Psychiatry and Behavioral Sciences, Baylor College of Medicine, Houston, TX, USA

${ }^{3}$ Departments of Radiology, Psychiatry and Biophysics, Weill Medical College of Cornell University, New York, NY, USA

${ }^{4}$ Department of Neuroscience, Mount Sinai School of Medicine, New York, NY, USA

${ }^{5}$ Comprehensive NeuroScience Inc., White Plains, NY, USA

\section{Edited by:}

Lisa M. Renzi, The University of Georgia, USA

\section{Reviewed by:}

Paul M. Nealen, Indiana University of PA, USA

Vincent Schmithorst, Children's Hospital Medical Center, USA

\section{*Correspondence:}

Jeremy D. Coplan, Division of Neuropsychopharmacology,

Department of Psychiatry, State University of New York Downstate Medical Center, 450 Clarkson Avenue, Brooklyn, NY 11023, USA. e-mail:copstat00@aol.com
We have demonstrated in a previous study that a high degree of worry in patients with generalized anxiety disorder (GAD) correlates positively with intelligence and that a low degree of worry in healthy subjects correlates positively with intelligence. We have also shown that both worry and intelligence exhibit an inverse correlation with certain metabolites in the subcortical white matter. Here we re-examine the relationships among generalized anxiety, worry, intelligence, and subcortical white matter metabolism in an extended sample. Results from the original study were combined with results from a second study to create a sample comprised of 26 patients with GAD and 18 healthy volunteers. Subjects were evaluated using the Penn State Worry Questionnaire, the Wechsler Brief intelligence quotient (IQ) assessment, and proton magnetic resonance spectroscopic imaging ( $\left.{ }^{1} \mathrm{H}-\mathrm{MRSI}\right)$ to measure subcortical white matter metabolism of choline and related compounds $(\mathrm{CHO})$. Patients with GAD exhibited higher IQ's and lower metabolite concentrations of $\mathrm{CHO}$ in the subcortical white matter in comparison to healthy volunteers. When data from GAD patients and healthy controls were combined, relatively low $\mathrm{CHO}$ predicted both relatively higher IQ and worry scores. Relatively high anxiety in patients with GAD predicted high IQ whereas relatively low anxiety in controls also predicted high IO. That is, the relationship between anxiety and intelligence was positive in GAD patients but inverse in healthy volunteers. The collective data suggest that both worry and intelligence are characterized by depletion of metabolic substrate in the subcortical white matter and that intelligence may have co-evolved with worry in humans.

Keywords: intelligence, anxiety, white matter, choline, magnetic resonance spectroscopic imaging

\section{INTRODUCTION}

Humans are the most intelligent species and also appear to suffer disproportionately from psychopathology. One parameter that potentially contributes to both intelligence and psychopathology is that of "connectivity" between neurons, which putatively enhances neural processing capacity independent of brain size (Jerison, 1977). The white matter of the brain, which contains tracts of myelinated axons, is dedicated solely to facilitating this connectivity. It appears likely that white matter, though devoid of neuronal cell bodies, participates in neural processing through axonal gap junctions (Hamzei-Sichani et al., 2007). Several studies have noted a positive correlation between white matter volume and intelligence at the level of whole brain white matter volume as well as in specific white matter regions (for example, see Haier et al., 2004). Schoenemann et al. (2005) have furthermore suggested that white matter plays an important role in the evolution of the human brain by demonstrating that prefrontal white matter volume is disproportionately large in humans compared to non-human primates. It should be noted, however, that the conclusions reached by the authors regarding white matter volume differences were subsequently questioned (Sherwood et al., 2005).

In a previous study (Coplan et al., 2006), we explored associations among generalized anxiety, worry, intelligence, and brain metabolite concentrations within the centrum semiovale (CSO) white matter. The CSO was selected as a region of interest (ROI) because it comprises the central white matter core of the cerebral hemispheres. Tissue concentrations of three metabolites $-\mathrm{N}$ acetyl-aspartate (NAA), a putative marker of neuronal integrity or density; choline-containing compounds $(\mathrm{CHO})$, reflective of membrane turnover; and creatine + phosphocreatine (CR), historically used as a stable measure for MRS ratio analyses - were quantified using proton magnetic resonance spectroscopic imaging $\left({ }^{1} \mathrm{H}-\mathrm{MRSI}\right)$. Whereas previous studies had positively linked prefrontal cortex NAA concentrations to intelligence in healthy subjects (Valenzuela et al., 2000; Pfleiderer et al., 2004; Jung et al., 2005; Ross et al., 2005), we did not find a relationship between CSO NAA concentrations and intelligence quotient (IQ; Hays et al., 2002). Nor did we detect a relationship between CSO NAA concentrations and worry. We did, however, demonstrate that both 
worry and IQ correlate inversely with choline-containing compounds in the white matter CSO. It had previously been posited that low $\mathrm{CHO}$ represents a depletion of metabolic substrate (Dawson et al., 1985; Duc et al., 1997). Thus the data were interpreted to suggest that both high IQ and high worry are characterized by enhanced metabolism of the white matter CSO.

In the same study, the association between IQ and worry was especially intriguing. The relationship between intelligence and anxiety has been heavily explored. As far back as Spielberger (1958) noted a negative correlation between intelligence and anxiety in healthy subjects. This relationship has been explained as follows: individuals exhibiting low intelligence are unable to make responses that lead to success, which in turn results in the development of anxiety (Phillips et al., 1960). Others have suggested a positive correlation between intelligence and pathological anxiety based on the hypothesis that gifted children are prone to disharmonious development, which may result in the development of personality disorders, obsessional behavior, and anxiety disorders. According to Catheline-Antipoff and Poinso (1994), "the higher the IQ is, the greater the psychological fragility." In our study, healthy volunteers exhibited an inverse relationship between IQ and worry. In contrast, in patients with DSM-IVdiagnosed generalized anxiety disorder (GAD; First et al., 1995), IQ correlated positively with worry scores on the Penn State Worry Questionnaire (PSWQ; Behar et al., 2003). Thus, high intelligence was associated with either the least or greatest degree of worry, depending on whether or not the subject carried a diagnosis of GAD.

Several factors limited the interpretability of the study results. First, the original sample size was small $(n=15$ for the control group and $n=15$ for the GAD group). The sample size was further reduced after two subjects from the GAD group were excluded (one patient exhibited an IQ that was $>4$ SDs outside the group mean and IQ scores were missing for one patient). Additionally, six subjects in the GAD group who had experienced early trauma were excluded from analysis of CSO white matter metabolism after it was noted that the presence of early trauma significantly modified the MRSI metabolite profile of GAD patients. Further, recruitment bias may have resulted in a sample of GAD patients that exhibited significantly higher intelligence in comparison to the sample of healthy volunteers.

In order to further explore the association between salutary human development (i.e., intelligence) and psychiatric symptomatology, we re-examine the relationships among generalized anxiety, worry, intelligence, and brain metabolite concentrations within the CSO white matter in an extended sample of patients with GAD and healthy controls.

\section{MATERIALS AND METHODS SUBJECTS}

Eighteen healthy volunteers (eight males and 10 females) and 26 patients with GAD (12 males and 14 females) served as subjects. The sample comprised patients from the original, abovementioned study (Study \#1; Coplan et al., 2006) with the addition of patients from a second study (Study \#2; Mathew et al., 2008). From the original study, this represents an increase in sample size of $20 \%$ for the control group and $73 \%$ for the GAD group (and an increase of $100 \%$ for the GAD group when excluding subjects with early trauma).

Non-psychiatrically ill controls were required to have a negative family history in first-degree relatives, and were matched for handedness in the first study (all were right-handed) and three patients and one control were left-handed for the second study. As determined by the Structured Clinical Interview for DSM-IV Axis I Disorders as described by First et al. (1995), comorbid disorders in the GAD group were dysthymia $(n=9)$, social anxiety disorder $(n=3)$, panic disorder with or without agoraphobia $(n=6)$, and concurrent social anxiety disorder with dysthymia $(n=1)$. Inclusion criteria required that GAD precede onset of comorbid Axis I Disorders. Exclusion criteria were a major depressive episode or substance abuse within 6 months of study entry; a lifetime history of psychotic, bipolar, obsessive-compulsive, post-traumatic stress, or eating disorder; substance dependence (other than nicotine); mental retardation or learning disability; autism; or significant medical or neurological conditions. Of the GAD patients, 21 were medication-naive, and no subject had psychotropic exposure at least 4 weeks before scanning. Symptom severity, for the purposes of the current study, was measured at the time of the MRSI scan by the PSWQ. In order to evaluate the potential contribution of early life physical and sexual abuse to MRSI measures, subjects were systematically assessed using a modified version of the self-report early trauma inventory (ETI; Bremner et al., 2000). Eight of 26 $(31 \%)$ of our GAD patients had suffered severe prepubertal physical and/or sexual abuse but had not developed post-traumatic stress disorder (PTSD). The Wechsler abbreviated scale of intelligence (WASI), a brief IQ test, with high concordance rates to other IQ tests (Hays et al., 2002), was applied to all participants. Although an IQ $<70$ was an exclusion criteria for participation in the studies, one patient subject with GAD had an IQ of 71 which was $>4$ SD outside the total IQ mean and was excluded. All subjects gave written signed informed consent after a complete verbal and written explanation of the procedures, and the protocol was approved by the Institutional Review Boards of Columbia University and the New York State Psychiatric Institute and Mount Sinai Medical Center.

\section{${ }^{1} \mathrm{H}$-MRSI DATA ACOUISITION}

Scans were performed on two separate 1.5 T GE Signa scanners, using the multi-slice ${ }^{1} \mathrm{H}$-MRS imaging sequence (Duyn et al., 1993) as previously described (Mathew et al., 2004). Following Sagittal scout images, a four-section T1-weighted axial/oblique localizer image, angulated parallel to the Sylvian fissure, was acquired, with a slice thickness of $15 \mathrm{~mm}$ and an inter-slice gap of $3.5 \mathrm{~mm}$, matching the subsequent ${ }^{1} \mathrm{H}$-MRS scan. Next, a multi-slice MRSI scan was performed, which is a standard slice-interleaved spin echo sequence, except that it uses octagonally tailored outer volume presaturation pulses to suppress the strong lipid and fat signals from the scalp, skull, and calvarial marrow. Water suppression was achieved with a water-selective radiofrequency pulse followed by strong spoiler gradients. Since the major metabolite of interest [CHO] has a relatively long transverse relaxation times in the main ROI, a long echo time $(\mathrm{TE}=280 \mathrm{~ms})$ was used to minimize baseline distortion and spectral overlap, thereby facilitating fitting. The other acquisition 
parameters were: $\mathrm{TR}=2300 \mathrm{~ms}, \mathrm{FOV}=240 \mathrm{~mm}, 32 \times 32$ phaseencoding steps with circular $k$-space sampling, and 256 points along the signal acquisition domain. The resulting nominal MRSI voxel sizes were $1.5 \mathrm{~cm} \times 0.75 \mathrm{~cm} \times 0.75 \mathrm{~cm}$, or $0.84 \mathrm{cc}$.

\section{${ }^{1}$ H-MRSI DATA ANALYSIS}

The MRSI data were transferred to an off-line workstation for processing and analysis by two of the study investigators, who used interactive analysis software of their own design. A single researcher blinded to diagnosis selected the ROI's and performed spectral analysis. Another investigator, Dikoma C. Shungu, who is the senior spectroscopist, ensured that the analyses were performed properly but was not actively involved in ROI selection or data analysis. The raw data were sorted by slice, zerofilled twice along the acquisition domain (to 1024 sampling points), filtered with a Hamming window along spatial domains, and then processed by standard three-dimensional fast Fourier transformation to yield an array of $32 \times 32$ spectra. The spectral data were automatically corrected for susceptibility shifts due to slight variations in magnetic field strength across the brain using a computer routine that aligned the spectra by maximizing the cross correlation between a spectrum in the data matrix and a representative high SNR reference spectrum selected from the same data matrix. MRSI voxels that best covered the primary ROIs (right and left CSO) in each subject were selected on the basis of voxel location on the matching high-resolution MR localizer images (Figure 1). In each voxel, the $\mathrm{CHO}$ metabolite peak area was obtained using a frequencydomain Levenberg-Marquardt non-linear least-squares fitting algorithm. As many pixels as possible were fitted within each ROI and then averaged. Voxels with poor spectral resolution (unresolved CHO peaks at $3.22 \mathrm{ppm}$ ) were excluded. The number of voxels rejected varied from subject to subject and did not result in any patient being rejected. The mean of the peak areas for $\mathrm{CHO}$ and metabolites within an ROI was computed.

\section{ABSOLUTE METABOLITE QUANTITATION}

Mean regional peak areas derived from spectral fitting were converted to absolute metabolite concentrations using the phantom replacement methodology (Soher et al., 1996), which has been shown to give values that are in excellent agreement with the literature (Kreis et al., 1993). For further discussion of this quantification method, see Mathew et al. (2008, 2010). A Microsoft Excel worksheet was developed for facilitating the derivation of absolute $\mathrm{CHO}$ metabolite concentrations from spectral peak areas. The user simply inputted the mean regional peak area values and the appropriate spectral intensity correction parameters for both the phantom and metabolites, and the software calculated and tabulated the corresponding absolute metabolite concentrations.

\section{STATISTICAL ANALYSES}

Independent $t$-test compared GAD patients versus healthy control subjects for mean choline, IQ, and PSWQ. Only values for mean choline (and not values for right and left $\mathrm{CHO}$ ) were available. Sex, weight, age, and study (Study \#1 versus Study \#2) distributions were examined for group differences and, where appropriate, were controlled for. Pearson partial correlation matrices examined the relationship between IQ, mean choline, and PSWQ in all subjects. GAD patients with trauma were excluded from this analysis as trauma has been shown to produce an effect on mean choline for subcortical white matter which can be distinguished from GAD without early trauma (Coplan et al., 2006). To integrate the inter-correlations between IQ, PSWQ, and mean CHO into a single analysis, a Friedman's ANOVA and the Kendall coefficient of concordance were employed.

To examine the relationship between IQ and PSWQ as a function of group assignment, a general linear model was employed using a factorial design with group as a categorical variable, IQ as a continuous predictor variable and the interaction between group and IQ as the interactive variable. GAD plus trauma subjects were included in this analysis because trauma has not been shown to affect IQ or worry score as it does CHO metabolism (Coplan et al., 2006).

\section{RESULTS \\ ANALYSIS OF INDEPENDENT VARIABLES IN GAD PATIENTS AND HEALTHY VOLUNTEERS}

No significant group effect was observed for age [GAD group mean $=36.5 \pm 12.6$ years $(\mathrm{SD})$; Healthy Volunteer group mean $=38.0 \pm 13.2$ years $]$, weight $[\mathrm{GAD}$ group mean $=159.3 \pm$
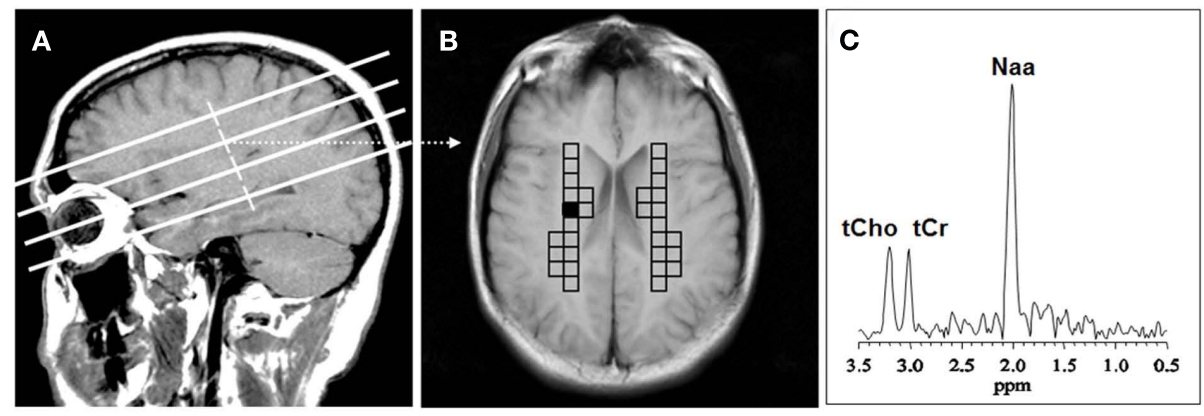

FIGURE 1 | Voxel placement for centrum semiovale (CSO) white matter; (A) MRI depicting the location of the four oblique brain sections investigated; (B) Coronal view from second oblique section overlaid with left and right grids of CSO voxels of interest. (C) MR spectrum from the shaded CSO voxel in the grid shown in (B). NAA, N-acetyl-aspartate; tCho, total choline containing compounds; $\mathrm{tCr}$, total creatine + phosphocreatine. 
$39.1 \mathrm{lb}$ (SD); Healthy Volunteer group mean $=151.2 \pm 42.0 \mathrm{lb}]$, or sex and these were not used as control variables in partial correlation analyses (see Tables 1 and 2). Although the two groups did not differ with respect to age, because there was a difference in age distribution between the two studies [Study $\# 1$ mean $=40.0 \pm 13.4$ years; Study \#2 mean $=32.1 \pm 9.9$ years; $F(1,41)=4.08 ; p=0.04]$, study number was used as a covariate. Also, because mean choline was noted to increase with increasing age ( $r=0.37 ; N=44 ; p=0.012)$, age was used as a covariate. Patients with GAD reported significantly more worry, exhibited higher IQ scores, and showed reduced concentrations of cholinecontaining compounds in the subcortical white matter voxels (see Table 1).

\section{CORRELATIONS AMONG MEAN CHOLINE, I0, AND WORRY}

Controlling for age and study, analyses revealed significant inverse correlations between mean choline and both IQ and PSWQ scores (see Table 3). The combined effect for these inter-correlations was highly significant [Friedman ANOVA Chi Square $(N=36, \mathrm{df}=2)=72.00 ; p<0.00001$; Kendall Coeff. of Concordance $=1.0000$ Aver. rank $r=1.0000$ ].

There were no significant group $\times$ choline interactions for either IQ or PSWQ when covarying for age and study. For PSWQ, the group $\times$ choline interaction was $[F(1,36)=2.17 ; p=0.15]$. For IQ, a trend for a group $\times$ choline interaction was observed $[F(1,36)=3.76 ; p=0.06]$. Upon inspection of the individual groups' correlations between mean choline and IQ; in GAD patients a very weak non-significant inverse correlation was noted $(r=-0.13 ; p=0.6)$ whereas for the healthy volunteers a nonsignificant positive correlation was noted $(r=0.38 ; p=0.13)$. As the correlations were non-significant in both groups, and the

Table 1 | Comparison of means and SDs for patients with generalized anxiety disorder versus healthy volunteers.

\begin{tabular}{lccccl}
\hline & GAD $(\boldsymbol{N}=\mathbf{2 6})$ & $\mathbf{H V}(\boldsymbol{N}=\mathbf{1 8})$ & $\boldsymbol{t}$-Value & $\mathbf{d f}$ & $\boldsymbol{p}$ \\
\hline Age (years) & $36.54 \pm 12.55$ & $38.00 \pm 13.19$ & -0.37 & 42 & 0.712 \\
Weight (Ib) & $159.27 \pm 39.09$ & $151.19 \pm 42.00$ & 0.65 & 42 & 0.517 \\
IQ & $118.65 \pm 9.31$ & $109.44 \pm 14.28$ & 2.59 & 42 & $\mathbf{0 . 0 1 3}$ \\
PSWO & $64.38 \pm 8.64$ & $30.06 \pm 8.27$ & 13.18 & 42 & $\mathbf{0 . 0 0 0}$ \\
Mean choline & $1.30 \pm 0.24$ & $1.47 \pm 0.19$ & -2.55 & 42 & $\mathbf{0 . 0 1 5}$ \\
\hline
\end{tabular}

$G A D$, generalized anxiety disorder patients; $H V$, healthy volunteers; $d f$, degrees of freedom; IQ, intelligence quotient; PSWQ, Penn State Worry Questionnaire.

Table 2 |Two-by-two tables comparing distributions between sexes for diagnostic groups.

\begin{tabular}{lrcc}
\hline & HV & GAD & Sex \\
\hline Male & 8 & 12 & 20 \\
Female & 10 & 14 & 24 \\
Group & 18 & 26 & 44 \\
\hline
\end{tabular}

$H V$, healthy volunteers; $G A D$, generalized anxiety disorder, Chi-squared $=0.01$, $p=0.9109$ group $\times$ choline interaction was a trend, there is not sufficient grounds to make an interpretation of this data. However, when the groups are combined, significant effects, which are presented in Table 3, are observed.

\section{RELATIONSHIP BETWEEN IO AND WORRY}

The groups differed with respect to the relationship between IQ and worry scores. In GAD patients, high IQ was associated with a greater degree of worry $(r=0.46 ; p=0.016)$. In healthy volunteers, high IQ was associated with a relatively lower degree of worry $(r=-0.60 ; p=0.009)$. The correlation between IQ and worry was significant in both the GAD group and the healthy control group. However, in the former, the correlation was positive and in the latter, the correlation was negative (see Figure 2). Effects remained significant when covarying for age and study number.

A full factorial general linear design with group and IQ as predictor variables and PSWQ score as the dependent variable revealed an interaction between group and IQ in predicting PSWQ score (see Table 4).

\section{DISCUSSION}

Results from the original study were reported with the limitation that the sample size was small and that the IQ scores may have been spuriously elevated in the GAD group compared to the control group. In this extended study, the sample size has been increased, thus making these results more robust than those from the original study. It should be noted, however, that even with the increased sample size, the mean IQ for the GAD group was elevated in comparison to the control group (a mean of $\sim 119$ versus a mean of 109). Further, the IQ means for both the GAD group and the control group were significantly higher than the average IQ of the general population $(\sim 100)$. Results should thus be interpreted with the consideration that the study sample is non-representative of the general population.

It should furthermore be noted that differences in the neural substrates of intelligence have been observed in men and women (Haier et al., 2005). In our study, male and female subjects were combined into a single analysis in order to increase sample size, and this is a potential limitation of the study. The nature of sex differences as they relate to intelligence, anxiety, and white matter metabolism should be characterized in future studies.

Table 3 | Partial correlation matrix analyzing for relationship between IO, worry, and centrum semiovale white matter choline; GAD plus early trauma excluded, age and study number used as covariates.

\begin{tabular}{llll}
\hline & Mean choline & IQ & PSWQ \\
\hline Mean choline & 1.0000 & & \\
& $p=-$ & & \\
IQ & -0.5620 & 1.0000 & \\
PSWO & $\boldsymbol{p}=\mathbf{0 . 0 0 1}$ & $p=-$ & 1.0000 \\
& -0.4223 & 0.1777 & $p=-$ \\
\hline
\end{tabular}

1Q, intelligence quotient; PSWQ, Penn State Worry Questionnaire. 


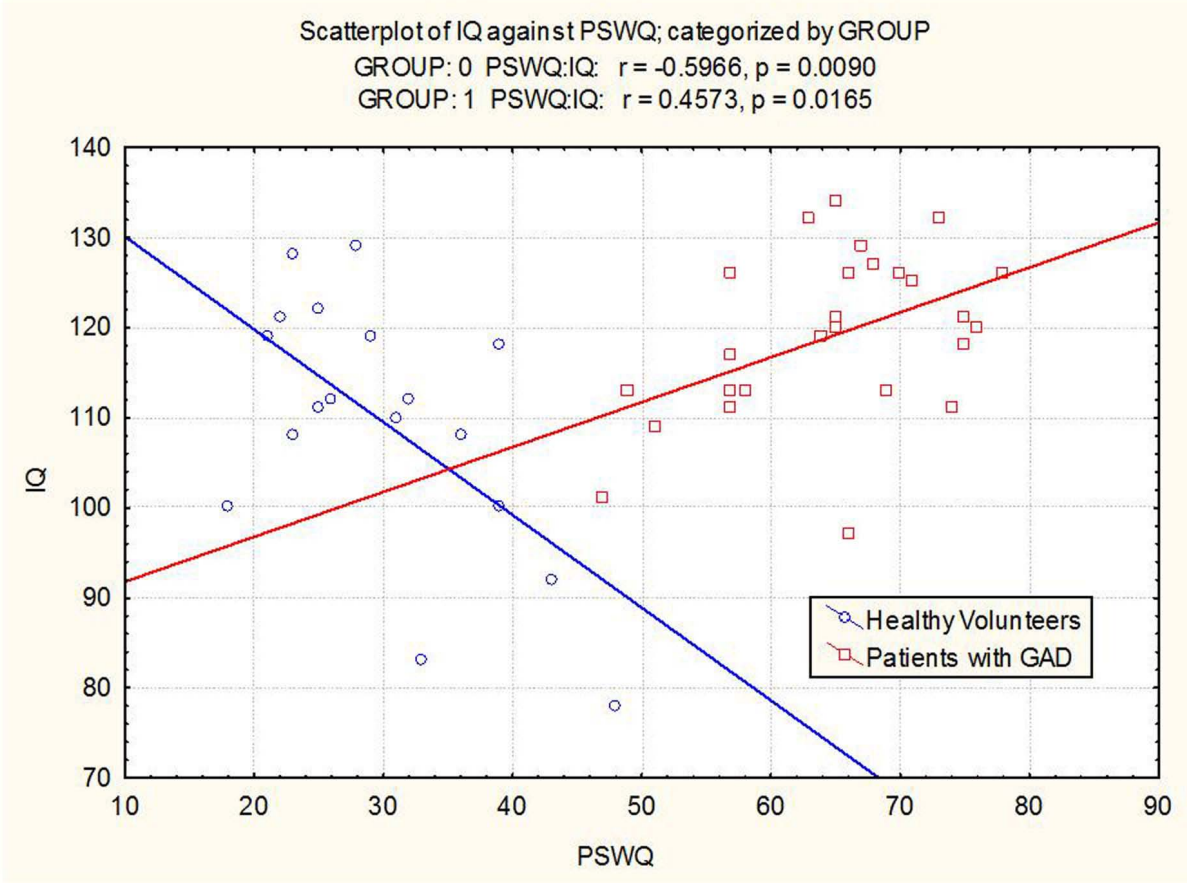

FIGURE 2 | Scatterplot of 10 versus worry in patients with GAD and healthy volunteers (Group $0=$ healthy volunteers, Group $1=$ GAD patients).

Table 4 | Full factorial general linear design with group (GAD versus $\mathrm{HV}$ ) and $\mathrm{IO}$ as predictor variables and worry scores as the dependent variable.

\begin{tabular}{llrl}
\hline & Degr. of freedom & $\boldsymbol{F}$ & $\boldsymbol{p}$ \\
\hline Group & $1 ; 39$ & 6.65 & $\mathbf{0 . 0 1 3 8}$ \\
IQ & $1 ; 39$ & 0.14 & 0.7075 \\
Group*IQ & $1 ; 39$ & 16.45 & $\mathbf{0 . 0 0 0 2}$ \\
Study & $1 ; 39$ & 3.30 & 0.0770 \\
Age & $1 ; 39$ & 0.09 & 0.7643
\end{tabular}

$G A D$, generalized anxiety disorder; $H V$, healthy volunteers; 10 , intelligence quotient, Degr., degrees, Worry scores measured by the Penn State Worry Questionnaire, Study Number, and Age used as control variables.

Another potential limitation of the study lies in the collection of IQ data. Only Full-scale IQ, and not the sub-indices of Verbal IQ or Performance IQ (including Perceptual Organization), was measured. It is possible that the various IQ sub-indices correlate uniquely with worry and with levels of white matter $\mathrm{CHO}$. This issue should be further investigated.

With these caveats in mind, in this extended sample, we have replicated our results tying human intelligence to pathological worry (Coplan et al., 2006). Patients with generalized anxiety exhibited, along with the expected increase in self-reported worry, relatively high IQ scores and lower white matter choline and choline-containing compounds in comparison to healthy volunteers. When considering all subjects except those with early life trauma, white matter $\mathrm{CHO}$ concentrations correlated inversely with both IQ and worry scores. We have previously hypothesized that low white matter $\mathrm{CHO}$ represents a depletion of metabolic substrate (Dawson et al., 1985; Duc et al., 1997). As such, subcortical white matter metabolic activity may represent a functional neuro-evolutionary commonality for both intelligence and worry. That is, one region of the brain - the white matter - that has contributed to the evolutionary success of humans may also be implicated in anxiety disorders.

Pathological anxiety has heretofore been considered maladaptive: it represents a response to distal or potential threats that are deemed either entirely symbolic or extremely unlikely to present actual danger to the individual (for a review, see Grillon, 2008). But, if high anxiety is maladaptive, then it should not correlate positively with intelligence, the most adaptive trait for humans, as we have shown in this study. With respect to humans exhibiting high intelligence in this sample, we have demonstrated the presence of two distinct phenotypes: individuals diagnosed with GAD showing a high degree of pathological worry and healthy individuals exhibiting a low degree of worry. So, we propose that the socially based evolution of humans may actually favor the expression of alleles responsible for high anxiety and worry. While an intelligent, non-anxious person can assess that a distal threat is exceedingly remote, the worrying individual is adapted to never entirely abandon the possibility of danger and, as a result, may adopt certain avoidance behavioral patterns. It is possible that adaptations that favor involuntary imposition of such avoidance patterns may limit potentially dangerous social contact or situations (Gilbert, 2001), and the induction of anxious cognitions of impending catastrophe may thereby represent a viable human phenotype. 
The results of our study call to mind recent research done by Cochran et al. (2006) in which investigators concluded that the social environment in medieval Europe allowed for the natural selection of high intelligence in the Ashkenazi Jewish population. That is, the restriction of Jews to managerial and commerce professions that required intellectual acumen created a high fitness payoff to increased verbal and mathematical intelligence, and, as a result, the intelligence of the Ashkenazi population increased over the ensuing 1000 years. Researchers further proposed that the genetic mutations in sphingolipids and DNA repair that, in their homozygous forms, result in devastating diseases such as TaySachs may actually promote neuronal growth and connectivity in heterozygous carriers thereby increasing intelligence.

In conclusion, we are able to demonstrate activation of a common neural substrate, the subcortical white matter, which varies parametrically with both anxiety and worry in a positive manner. This suggests that what we have labeled as

\section{REFERENCES}

Behar, E., Alcaine, O., Zuellig, A. R., and Borkovec, T. D. (2003). Screening for generalized anxiety disorder using the Penn State Worry Questionnaire: a receiver operating characteristic analysis. J. Behav. Ther. Exp. Psychiatry 34, 25-43.

Bremner, J. D., Vermetten, E., and Mazure, C. M. (2000). Development and preliminary psychometric properties of an instrument for the measurement of childhood trauma: the early trauma inventory. Depress. Anxiety 12, 1-12.

Catheline-Antipoff, N., and Poinso, F. (1994). Gifted children and dysharmonious development. Arch. Pediatr. 1, 1034-1039.

Cochran, G., Hardy, J., and Harpending, H. (2006). Natural history of Ashkenazi intelligence. J. Biosoc. Sci. 38, 659-693.

Coplan, J. D., Mathew, S. J., Mao, X., Smith, E. L., Hof, P. R., Coplan, P. M., Rosenblum, L. A., Gorman, J. M., and Shungu, D. C. (2006). Decreased choline and creatine concentrations in centrum semiovale in patients with generalized anxiety disorder: relationship to IQ and early trauma. Psychiatry Res. 147, 27-39.

Dawson, R. M., Hemington, N., and Irvine, R. F. (1985). The inhibition of diacylglycerol-stimulated intracellular phospholipases by phospholipids with a phosphocholine-containing polar group. A possible physiological role for sphingomyelin. Biochem. J. 230, 61-68.

Duc, C. O., Weber, A. H., Trabesinger, A. H., Meier, D., and Boesiger, P. (1997). Recycling the cholines. Int. Soc. Magn. Reson. Med. 6, 1210.
Duyn, J. H., Gillen, J., Sobering, G., van Zijl, P. C., and Moonen, C. spectroscopic imaging of the brain. Radiology 188, 277-282.

First, M. B., Spitzer, R. L., Gibbon, M., and Williams, J. B. W. (1995). Structured Clinical Interview for DSMIV Axis I Disorders, Patient Edition (SCIDP), Version 2. New York: Biometrics Research, New York State Psychiatric Institute.

Gilbert, P. (2001). Evolution and social anxiety. The role of attraction, social competition, and social hierarchies. Psychiatr. Clin. North Am. 24, 723-751.

Grillon, C. (2008). Models and mechanisms of anxiety: evidence from startle studies. Psychopharmacology (Berl.) 199, 421-437.

Haier, R. J., Jung, R. E., Yeo, R. A., Head, K., and Alkire, M. T. (2004). Structural brain variation and general intelligence. Neuroimage 23, 425-433.

Haier, R. J., Jung, R. E., Yeo, R. A., Head, K., and Alkire, M. T. (2005). The neuroanatomy of general intelligence: sex matters. Neuroimage 25, 320-327.

Hamzei-Sichani, F., Kamasawa, N., Janssen, W. G., Yasumura, T., Davidson, K. G., Hof, P. R., Wearne, S. L., Stewart, M. G., Young, S. R., Whittington, M. A., Rash, J. E., and Traub, R. D. (2007). Gap junctions on hippocampal mossy fiber axons demonstrated by thin-section electron microscopy and freeze fracture replica immunogold labeling. Proc. Natl. Acad. Sci. U.S.A. 104, 12548-12553.

Hays, J. R., Reas, D. L., and Shaw, J. B. (2002). Concurrent validity T. (1993). Multisection proton MR

maladaptive - pathological anxiety - co-evolved with the attribute that is viewed as most adaptive, human intelligence.

\section{ACKNOWLEDGMENTS}

This research was supported by GlaxoSmithKline Pharmaceuticals, Sackler Institute of Columbia University, National Institute of Mental Health Grant MH59990 and National Alliance for Research on Schizophrenia and Depression Mid-Investigator Award (Jeremy D. Coplan); National Alliance for Research on Schizophrenia and Depression Young Investigator's Award (Sanjay J. Mathew), Psychiatric Institute Research Support Grant and National Institute of Mental Health research fellowship T32 MH15144 (Sanjay J. Mathew); and Career Development Award NIMH (K23 MH069656) (Sanjay J. Mathew). We thank Jonathan Amiel, M.D., Steven Dashnaw, Heidi Fitterling, M.P.H., Joy Hirsch, Ph.D., Kathryn Keegan, and Harold Sackeim, Ph.D., for their contributions.

of the Wechsler abbreviated scale of intelligence and the Kaufman brief intelligence test among psychiatric inpatients. Psychol. Rep. 90, 355-359.

Jerison, H. (1977). The theory of encephalization. Ann. N. Y. Acad. Sci. 299, 146-160.

Jung, R. E., Haier, R. J., Yeo, R. A., Rowland, L. M., Petropoulos, H., Levine, A. S., Sibbitt, W. L., and Brooks, W. M. (2005). Sex differences in Nacetylaspartate correlates of general intelligence: an 1H-MRS study of normal human brain. Neuroimage 26, 965-972.

Kreis, R., Ernst, T., and Ross, B. D. (1993). Development of the human brain: in vivo quantification of metabolite and water content with proton magnetic resonance spectroscopy. Magn. Reson. Med. 30, 424-437.

Mathew, S. J., Price, R. B., Shungu, D. C., Mao, X., Smith, E. L., Amiel, J. M., and Coplan, J. D. (2010). A pilot study of the effects of chronic paroxetine administration on hippocampal N-acetylaspartate in generalized anxiety disorder. J. Psychopharmacol. 24, 1175-1181.

Mathew, S. J., Mao, X., Coplan, J. D., Smith, E. L., Sackeim, H. A., Gorman, J. M., and Shungu, D. C. (2004). Dorsolateral prefrontal cortical pathology in generalized anxiety disorder: a proton magnetic resonance spectroscopic imaging study. Am. J. Psychiatry 161, 1119-1121.

Mathew, S. J., Price, R. B., Mao, X. Smith, E. L., Coplan, J. D., Charney, D. S., and Shungu, D. C. (2008). Hippocampal $\mathrm{N}$-acetylaspartate concentration and response to riluzole in generalized anxiety disorder. Biol. Psychiatry. 63, 891-898.

Pfleiderer, B., Ohrmann, P., Suslow, T., Wolgast, M., Gerlach, A. L., Heindel, W., and Michael, N. (2004). Nacetylaspartate levels of left frontal cortex are associated with verbal intelligence in women but not in men: a proton magnetic resonance spectroscopy study. Neuroscience 123, 1053-1058.

Phillips, B. N., Hindsman, E., and Jennings, E. (1960). Influence of intelligence on anxiety and perception of self and others. Child Dev. 31, 41-46.

Ross, A. J., Sachdev, P. S., Wen, W., Valenzuela, M. J., and Brodaty, H. (2005). Cognitive correlates of $1 \mathrm{H}$ MRS measures in the healthy elderly brain. Brain Res. Bull. 66, 9-16.

Schoenemann, P. T., Sheehan, M. J., and Glotzer, L. D. (2005). Prefrontal white matter volume is disproportionately larger in humans than in other primates. Nat. Neurosci. 8, 242-252.

Sherwood, C. C., Holloway, R. L., Semendeferi, K., and Hof, P. R. (2005). Is prefrontal white matter enlargement a human evolutionary specialization? Nat. Neurosci. 8, 537-538.

Soher, B. J., van Zijl, P. C., Duyn, J. H., and Barker, P. B. (1996). Quantitative proton MR spectroscopic imaging of the human brain. Magn. Reson. Med. 35, 356-363.

Spielberger, C. D. (1958). On the relationship between manifest anxiety and intelligence. J. Consult. Psychol. 22, 220-224.

Valenzuela, M. J., Sachdev, P. S., Wen, W., Shnier, R., Brodaty, H., and 
Gillies, D. (2000). Dual voxel proton magnetic resonance spectroscopy in the healthy elderly: subcorticalfrontal axonal $\mathrm{N}$-acetylaspartate levels are correlated with fluid cognitive abilities independent of structural brain changes. Neuroimage 12, 747-756.

Conflict of Interest Statement: Dr. Coplan receives grant support from
GlaxoSmithKline and Pfizer pharmaceuticals, and he is on the Pfizer advisory board and gives talks for BMS, AstraZeneca, GlaxoSmithKline, and Pfizer. Dr. Gorman is an employe of Comprehensive NeuroScience, Inc., a corporation that receives funding from the pharmaceutical industry for some of its projects. No biomedical financial interests or potential conflicts of interest are reported for
Drs Mathew, Hodulik, Mao, Hof, or Shungu.

Received: 16 May 2011; accepted: 30 November 2011; published online: 01 February 2012.

Citation: Coplan JD, Hodulik S, Mathew SJ, Mao X, Hof PR, Gorman JM and Shungu DC (2012) The relationship between intelligence and anxiety: an association with subcortical white matter metabolism. Front. Evol. Neurosci. 3:8 doi: 10.3389/fnevo.2011.00008

Copyright (C) 2012 Coplan, Hodulik, Mathew, Mao, Hof, Gorman and Shungu. This is an open-access article distributed under the terms of the Creative Commons Attribution Non Commercial License, which permits noncommercial use, distribution, and reproduction in other forums, provided the original authors and source are credited. 